\title{
Aspectos clínicos, patológicos e sorológicos da toxoplasmose experimental em cães jovens
}

\section{Clinical, pathological and serologic aspects of experimental toxoplasmosis in young dogs}

\author{
Cláudia Bonini de Abreu²; Italmar Teodorico Navarro ${ }^{3 *}$; Mara Regina S. Balarin ${ }^{3}$; \\ Ana Paula F. R. L. Bracarense ${ }^{3}$; Elizabete Regina Marangoni Marana ${ }^{4}$; Sílvia Manduca Trapp ${ }^{5}$; \\ Cristiane Ayumi Fuginaka ${ }^{5}$; Leonardo Begale Prudêncio ${ }^{6}$; Marcos Rodrigues Matos; \\ Vinícius Suehiro Tsutsui ${ }^{6}$
}

\begin{abstract}
Resumo: A toxoplasmose é causada pelo Toxoplasma gondii, que acomete todos os animais de sangue quente inclusive o homem. A falta de informação na literatura da resposta clínica dos cães à infecção pelo T. gondii levou-nos a inocular experimentalmente nove cães jovens com três diferentes cepas, onde foram acompanhados durante 30 dias. Nesse período observou-se aumento de volume dos linfonodos em todos os cães inoculados e alterações oftalmológicas em sete cães. Titulo de anticorpos anti-T.gondii observados já 4ํㅡㄹ DPI demonstraram a precocidade da resposta nestes animais. O T. gondii foi isioaldo no sangue e urina da todos os cães e a doença foi confirmada histopatologicamnete. Alterações sorológicas, hematológicas e bioquímicas observadas, foram compatíveis com infecção pelo T.gondi. Assim os achados clínicos e laboratoriais foram característicos da toxoplasmose em cães jovens.
\end{abstract}

Palavras-chave: toxoplasmose, Toxoplasma gondii, cães, sinais clínicos.

Abstract: Toxoplasmosis is a disease caused by Toxoplasma gondii, that affects all warm- blooded- animals including human beings. The lack of information about the clinical response of dogs to $T$. gondii infection led us to experimentally inoculate nine young dogs with three different strains of $T$. gondii. The animals were examine daily during 30 days. Lymph node enlargement was the only clinical alteration detected in all dogs. Ophthalmologic changes were observed in seven dogs. T. gondii was isolated in blood and urine in all dogs and the disease was confirmed histopathologically. Serologic, hematologic, and biochemical changes compatible with T. gondii infection were observed. Our findings characterize clinical and laboratory changes in toxoplasmosis in young dogs.

Key words: Toxoplasmosis, Toxoplasma gondii, dogs, clinical signals.

\section{Introdução}

A toxoplasmose é causada pelo Toxoplasma gondii (NICOLLE; MANCEAUX, 1909), doença que acomete várias espécies, incluindo os mamíferos, répteis e aves (SANCHIS, 1978).

O homem e os animais podem adquirir a doença após o nascimento, principalmente pelo consumo de carnes cruas ou mal cozidas, infectadas com cistos teciduais, bem como alimentos e águas contaminados pelos oocistos (SOUZA et al., 1987; VIDOTTO, 1990; BROOKS, 1992; NAVARRO et al., 1992; BÓBIC et al., 1996). Leite não pasteurizado, ovos, transfusões sangüíneas e transplante de órgãos são outras vias de transmissão citadas (DRESSEN, 1990; DUBEY, 1993).

Helley (1970) afirmou que a toxoplasmose pode produzir uma série de alterações clínicas nos seres huma- nos e em muitas espécies animais estudadas tais como encefalite, abortamentos, pneumonia, enterite, miosite e ocasionalmente, coriorretinite, sendo esta última freqüentemente associada à uveíte. Opperman (1971) e Sharma et al. (1973) descreveram em cães experimentalmente infectados, as seguintes alterações: hipertermia, distúrbios pulmonares, digestivos e enfartamentos ganglionares.

Da mesma forma Nesbit et al. (1981) e Suter et al. (1984) observaram paresia espástica de membro pélvico em cães naturalmente infectados por T. gondii. Sintomatologia nervosa caracterizada por paraplégica, ataxia e hemiparesia foram detectadas em cães naturalmente infectados (AVERILL; LAHUNTA, 1971; NELSON; COUTO, 1994).

O sinal mais comum, reconhecido clinicamente como manifestação da toxoplasmose adquirida, é a linfade-

\footnotetext{
${ }^{1}$ Projeto financiado pela CPG-UEL/CAPES/CNPq.

2 Professor na UNIMAR-SP/Mestrando de Sanidade Animal /CCA/ DMVP/Universidade Estadual de Londrina - PR.

${ }^{3 *}$ Professor do Depto. de Med. Vet. Preventiva /Universidade Estadual de Londrina - PR / Endereço para correspondência e pedidos de separata: CX. Postal 6001, 86050-970 Londrina - PR. E-mail: <italmar@uel.br>.

${ }^{4}$ Médico Veterinário/Mestre em Sanidade Animal /CCA/DMVP/Universidade Estadual de Londrina PR.

${ }^{5}$ Residente na Clínica Médica e Cirúrgica dos Animais de Companhia.

${ }^{6}$ Acadêmico do Curso do Curso de Medicina Veterinária / Bolsista CNPq.
} 
nopatia cervical assintomática e fadiga (REMINGTON; DESMONT, 1983; McCABE et al., 1987).

Na literatura consultada nesta pesquisa, foram escassas as citações de estudos experimentais abordando aspectos clínicos de cães infectados por este parasito, da mesma forma não foi encontrada referência sobre infecção de cães jovens.

Assim, o objetivo do trabalho foi avaliar, a infecção experimental de diferentes cepas do $T$.gondii em cães jovens, quanto aos sinais clínicos, análises clínicas e a correlação entre os títulos de anticorpos obtidos.

\section{Material e Métodos}

No presente trabalho foram utilizadas três cepas de T. gondii, com característica particulares quanto a patogenia e patogenicidade em camundongos. A escolha das cepas foi determinada pela falta de informações, na literatura, quanto a patogenicidade em cães jovens. Sendo as cepas denominadas de $\mathrm{RH}$, isolada de ser humano com característica à resposta aguda e a não cronificação, AS-28, isolada de camundongo e $\mathrm{P}$, isolada de cão, ambas com característica à cronificação formando cistos e a formarem oocistos quando inoculadas em gatos. As cepas RH e AS-28 foram mantidas, no Laboratório de Protozoologia do Departamento de Medicina Veterinária Preventiva da Universidade Estadual de Londrina (UEL), e a cepa P mantida junto ao Departamento de Patologia Veterinária da Faculdade de Ciências Agrárias e Veterinárias de Jaboticabal (FCAVJ - UNESP), através de sucessivas passagens em camundongos albinos.

Foram selecionados 12 cães, com idade aproximada de 30 dias sorologicamente negativos pela Reação de Imunofluorescência Indireta (RIFI) para T. gondii (CAMARGO, 1964). Estes animais foram mantidos em jaulas individuais, durante todo o período de adaptação (4 meses) recebendo vacinas, anti-helmínticos, água a vontade e ração comercial para filhotes. Foram ainda acompanhados com exames clínicos completo e laboratoriais.

Dois grupos de 3 animais, sendo que cada grupo foi inoculado, respectivamente, com $5 \mathrm{~mL}$ (contendo 1,8 $\mathrm{x}$ $10^{4}$ oocistos $\left./ \mathrm{mL}\right)$ das cepas $\mathrm{P}$ e $5 \mathrm{~mL}\left(1,6 \times 10^{4}\right.$ oocistos $/ \mathrm{mL}$ ) da cepa AS-28 via oral com uso de sonda nasoesofágica, representando a principal via de infecção em cães jovens, obtidos de fezes de gatos previamente inoculados com cisto destas cepas. O terceiro grupo de cães foi inoculado com $5 \mathrm{~mL}\left(1.9 \times 10^{7}\right.$ taquizoítos/ $\mathrm{mL}$ ) da cepa $\mathrm{RH}$ divididos, $2,5 \mathrm{~mL}$ via intraperitoneal e $2,5 \mathrm{~mL}$ intravenosa concomitantemente, para evitar o óbito dos animais visto que esta cepa é altamente patogênica e não formadora de cisto ou oocisto, obtidos de lavado peritoneal de camundongos previamente inoculados. O quarto grupo, controle, foi inoculado com solução salina 0,85\% estéril, sendo cada animal por uma das vias, oral, intraperitoneal e intravenosa.

Os exames clínicos foram realizados duas vezes ao dia, durante todo o período experimental (30 dias), que compreendiam avaliações clínicas, neurológicas e oftalmológicas capazes de detectar alterações inerentes à evolução clínica da toxoplasmose. Os soros dos animais na pré e na pós-inoculação, obtidos a cada 3 ou 4 dias durante todo o experimento, foram submetidos a sorologia para pesquisa de anticorpos lgG anti-T. gondii pela RIFI, segundo Camargo (1964).

Nas colheitas de sangue alíquota, eram acondicionadas em frascos apropriados com EDTA e heparina, e submetidas a hemograma e perfil bioquímico respectivamente: alanina aminotransferase (ALT), fosfatase alcalina (FA), creatinina, uréia, glicose, albumina, amilase, bilirrubina direta e indireta realizados de acordo com as técnicas convencionais de análise laboratoriais (KANEKO, 1989).

As amostras do líquor, colhido a cada 15 dias com os animais devidamente anestesiados, foram submetidas a análise físico-química e citológica.

Avaliação da parasitemia foi realizada em amostras de sangue conservadas em EDTA. Uma alíquota foi separada e centrifugada a $693 \mathrm{~g}$ durante 15 minutos para separação da camada leucocitária. Essa camada assim obtida, de cada amostra, foi inoculada via intraperitoneal, em grupos de três camundongos albinos adultos.

Durante o período de observação de 35 dias, foi colhido líquido intraperitoneal dos camundongos que apresentaram sintomatologia clínica e morreram, para pesquisa de taquizoítos através de microscopia óptica direta. Os sobreviventes foram sacrificados, em câmara saturada com éter, sangrados para obtenção de soro para pesquisa de anticorpos IgG anti- $T$. gondii através da RIFI. Os cérebros dos camundongos sacrificados foram macerados e examinados, em microscopia óptica direta, para se verificar a presença de cistos.

Amostras de urina de cada cão, colhidas na préinoculação e semanalmente durante o experimento foram submetidas a urinálise. Dez $\mathrm{mL}$ de urina de cada animal foi centrifugado à $1083 \mathrm{~g}$ por 15 minutos, adicionando-se ao sedimento um $\mathrm{mL}$ de solução salina contendo $500 \mathrm{UI}$ de penicilina e um mg de estreptomicina. Após repouso de uma hora a 4ํㅡ $\mathrm{C}$ as amostras foram inoculadas via intraperitoneal em grupos de três camundongos suíços albinos e acompanhados durante 35 dias, conforme procedimento descrito para avaliação da parasitemia.

Ao final do experimento, 30 dias após inoculação, os cães foram eutanásiados e submentidos a exames de necrópsia. Após a avaliação macroscópica, foram colhidos para exames histopatológicos e ensaio biológico em camundongos, fragmentos de olho, baço, cérebro, coração, fígado, linfonodos (mesentéricos, mandibulares, sub-ilíaco), medula espinhal, músculo, pulmão e rins. Amostras destes tecidos foram fixados em formol tamponado a $10 \%$.

Os cortes teciduais foram corados pelo método da hematoxilina-eosina (HE) e fragmentos do coração, linfonodos, medula espinhal, musculatura esquelética, pulmão e rins foram submetidos a digestão péptica 
(JACOBS; MELTON, 1957). Os demais órgãos dos cães foram macerados, ressuspensos em salina estéril a 0,85\% e adicionados de 2000 UI de penicilina e $200 \mathrm{mg} / \mathrm{mL}$ de estreptomicina. Esses produtos assim obtidos, foram inoculados, via intraperitonial, em grupos de três camundongos e observados durante 35 dias (DUBEY et al.,1995).

\section{Resultados}

No período que antecedeu a inoculação, nenhum sinal clínico foi observado nos animais. Após a inoculação o único sinal clínico observado foi o aumento de volume dos linfonodos mandibulares, que se manifestou em todos os cães inoculados com T. gondii.

Da mesma forma nenhum animal apresentou qualquer alteração neurológica nos testes aplicados, no entanto alterações no sistema ocular com áreas de hiperrefletividade, foram observadas em sete animais, sendo dois inoculados com a cepa AS-28; dois com a $P$ e três com a RH. Esses achados foram confirmados através de retinografia em três animais onde observaramse as seguintes alterações: edema de papila, edema perivascular temporal, exsudato peripapilar bilateral. Um cão apresentou tapeto hipopigmentado na porção anterior do olho esquerdo.

As alterações hematológicas observadas foram: leucocitose em um animal, leucopenia em três animais e linfopenia em um animal.

No perfil bioquímico realizado nos cães, a proteína total, albumina e glicose foram as únicas alteradas em todos os animais, inclusive no grupo controle. As análises de ALT, FA, amilase, uréia, creatinina, bilirrubina direta e indireta não apresentaram qualquer alteração, assim como o líquor.
Os resultados dos exames, obtidos através da RIFI, foram considerados positivos quando apresentaram título maior ou igual a 1:16 Ishizuka, et al. (1974 e 1981), Germano et al. (1985), GuimarãeS et al. (1992), os quais encontram-se na Tabela 1. Anticorpos IgG anti- $T$. gondii foram detectados nos cães 1, 2, 8 e 12 a partir do $4^{\circ}$ dia pós-infecção (DPI); nos cães 5,6 e 7 no $7^{\circ}$ D D $\mathrm{P}$ I, e no cão 3 a partir do $11^{\circ} \mathrm{DPI}$. Os maiores níveis de anticorpos séricos foram obtidos somente após o 14ำ DPI, com títulos máximos de 1:4096, nos cães inoculados com a cepa $\mathrm{RH}$. Não foram encontrados sororeagentes nos animais do grupo controle (Tabela 1).

Na Tabela 2 estão registradas a detecção direta de T. gondii em exsudato peritoneal e/ou cistos e indireta através da RIFI em camundongos inoculados com camadas leucocitárias e sedimento urinário dos cães dos quatro grupos experimentais. A presença do $T$. gondii foi comprovada na urina de quatro e no sangue de três dos cães inoculados.

Nos cães que foram necropsiados 30 dias após a inoculação com o T. gondii, foram observadas lesões macroscópicas, relacionadas no Quadro 1.

Alterações histopatológicas foram observadas em rim, pulmão e fígado de todos os cães e em baço e linfonodo de sete cães inoculados experimentalmente. Outras lesões estão relacionadas no Quadro 2.

Em pelo menos três amostras dos diferentes tecidos colhidos da cada cão foi possível isolar o parasita, sendo que do baço foi isolado em sete cães; da retina rim e cérebro em seis e da medula espinhal, músculo esquelético e do coração em cinco cães. Nas amostras colhidas do grupo controle não foi isolado o parasita (Tabela 3).

Tabela 1 - Títulos de anticorpos lgG anti-T. gondii à Reação de Imunofluorescência Indireta em cães jovens inoculados experimentalmente com 3 cepas do parasito. 
Tabela 2 - Achado histopatológico do globo ocular de cães jovens inoculados experimentalmente com três cepas de Toxoplasma gondii, Londrina PR.

Grupo: 1, 2, 3, - cepa AS 28.

Grupo: 4, 5, 6- cepa P.

Grupo: 7, 8, 9 - cepa RH.

Grupo: 10,11,12 - controle.

Quadro 1 - Achado anátomo-patológico em cães jovens inoculados experimentalmente com três cepas do $T$. gondii, 2000. 
Quadro 2 - Achados histopatológicos em cães jovens inoculados experimentalmente com três cepas de $T$. gondii , 2000. Londrina, PR.

Tabela 3 - Isolamento de Toxoplasma gondii em grupos de 3 camundongos inoculados com amostras de órgãos, oriundos de cães jovens inoculados experimentalmente com três cepas do parasito.

- : negativo

Ps : positiva na sorologia pela RIFI para Toxoplasmose (=1:64) em camundongos inoculados com órgãos dos cães.

Pt : presença de taquizoítos de T. gondii no exsudato de camundongos inoculados com órgãos dos cães.

Pc: presença de cistos de T. gondii nos encéfalos de camundongos inoculados com órgãos de cães.

\section{Discussão}

No estudo a linfadenopatia foi o único sinal clínico observado nos cães inoculados com as diferentes cepas, sendo que nenhuma sintomatologia foi observada nos cães do grupo controle. Da mesma forma, Lindsay et al. (1997) não observaram sinais clínicos em dois cães com um ano de idade, inoculados via oral com oocistos esporulados.

No entanto quando inocularam-se animais adultos e/ou prenhez, observaram-se sinais clínicos da toxoplas- mose como Bresciani (1997), que inoculou cadelas gestantes com $1,5 \times 10^{4}$ oocistos via oral e $1,0 \times 10^{7}$ taquizoítos via subcutânea, observou quadro agudo já no $7^{\circ}$ DPI, com lacrimejamento, corrimento nasal, aumento do murmúrio vesicular, taquicardia e hipertermia. $\mathrm{O}$ mesmo foi observado por Vidotto et al. (1987) em porcas prenhes e por Navarro et al. (1992) em suínos jovens.

Da mesma forma, na avaliação neurológica não foi possível detectar qualquer alteração que fosse sugestiva de infecção aguda pelo parasito. Esses resultados concordam inteiramente com Bresciani (1997), que 
também não observou qualquer alteração em animais adultos inoculados. A não apresentação de alterações neurológicas, pode ser devido ao curto período de observação dos animais aliado a o fato deste tipo de lesões necessitarem de tempo de evolução maior. No entanto alterações neurológico por toxoplasmose foram apresentados por Koestner e Cole (1960) e Nelson e Couto (1994) em cães com sinais de poliradiculoneurite, polimiosite e hiperextensão, e Averril et al. (1971) que relataram um quadro de paraplegia flácida e espástica com ataxia em quatro cães adultos.

Os exames de oftalmoscopia direta, assim como da retinografia dos cães, apresentaram resultados sem precedentes na literatura. Apenas Fialho (1953) observou alterações oculares em cães experimentalmente inoculados e Meric (1994) relata casos de um cães adultos com retinite e coroidite. No entanto na toxoplasmose humana, é comum a descrições de alterações oculares semelhantes as observados nos cães, como Dressen (1990) e Silveira (1997) que mostram casos que vão desde um simples edema de papila até necrose ocular gravíssimos com evolução para cegueira.

Todos os cães inoculados responderam ao estímulo imunogênico produzindo anticorpos anti- T. gondii já $4^{\circ}$ DPI em quatro animais, e alcançando títulos máximos por volta do 14ํaós a inoculação (Tabela 1). Os resultados reforçam a característica antigênica particular, de cada cepa do T. gondii nos hospedeiros. Dressen (1990) e Dubey et al. (1995) observaram que o fato de apresentarem maiores títulos de anticorpos não significa maiores lesões oriundo da presença de cistos, demonstrado nos achados do isolamento nos tecidos (Tabela 2).

Estes resultados harmonizam-se aos de Vidotto et al. (1987) em suínos e aos de Bresciani (1997) em cadelas, quanto ao dia inicial da resposta humoral e diferem dos de Costa et al. (1977) que observaram um período negativo de seis dias (RIFI) em bovinos experimentalmente infectados com T. gondii.

Os títulos sorológicos obtidos dentre os cães dos grupos I e II, que receberam oocistos e o grupo III, que recebeu taquizoítos, estão de acordo com levantamentos epidemiológicos em cães realizados por Ishizuka et al. (1981) e Navarro et al., (1992) que observaram níveis de anticorpos que oscilaram entre 1:16 à 1:4096, chegando em alguns casos até 1:8000. Justifica-se esses resultados quando cepas altamente imunogênicas e pouco cistogênicas levaram ao aparecimento de altos níveis de anticorpos sem contudo, desenvolverem da doença Dubey (1995). O grupo III caracterizado pela amostra $\mathrm{RH}$, demostrou esse fato, quando apresentou os mais altos títulos sorológicos, uma discreta clínica e o não isolamento do parasita em tecido ou formação de cistos.

Exames hematológicos e perfis bioquímicos da infecção toxoplásmica canina são bastante restritos na literatura, não permitindo assim, uma avaliação comparativa mais aprofundada com os parâmetros obtidos nesta pesquisa.
A necessidade de estudar a viabilidade de provas bioquímicas que possibilitem ou que auxiliem no diagnóstico de toxoplasmose canina, induziu a realização dos exames dessa natureza inseridos neste trabalho.

Apesar de autores como Roth et al. (1971), Hirt (1976), Costa et al. (1977), Kaneko (1989) afirmarem que o fígado possa ser um órgão muito afetado pela infecção do T. gondii, a pesquisa de níveis séricos de ALT, bilirrubina direta e indireta nos cães não apresentaram quaisquer alterações.

No entanto níveis elevados de albumina ( $8,5 \mathrm{~g} / \mathrm{dl}) \mathrm{e}$ proteína sérica total $(9,0)$ foram observados em todos animais (KANEKO, 1989), sugerindo quadro de desidratação, mesmo que momentânea, em virtude de calor excessivo.

Aumento dos valores de glicose sangüínea foram também observadas em todos os animais inoculados com T. gondii, inclusive grupo controle. Este fato sugere que essa alteração pode não estar associada a toxoplasmose mas sim ao confinamento e ao estresse ambiental, abstinência de água desses cães durante os exames, nas colheitas de material e na pré anestesia para algumas manobras clínicas (KANEKO,1989).

O achado do T. gondii na urina dos cães experimentais (Tabela 2) variou durante o período pós inoculação. O cão 7 eliminou o parasito no 6ำ dia, os cães 5, 6 e 8 no $13^{\circ}$ dia pós inoculação.

O isolamento na urina de quatro cães mostra a eliminação do $T$.gondii por essa via, sendo que em outros quatro animais foi isolado apenas no tecido renal. Esses achados sugerem a possibilidade desse órgão e da urina na disseminação dessa zoonose. Reforçando a importância dessa via de eliminação Vitor e Pinto (1991) estudando caprinos e Bresciani (1997) em cadelas prenhes, também isolaram o agente da urina.

Os achados anátomo-patológicos (Quadro 1) permitiram visualizar uma resposta do organismo frente a invasão do parasito, onde o timo, ativo em jovens, placas de Peyer e baço apresentaram-se alterados na maioria dos animais. Isso mostra um intenso estímulo do sistema mononuclear-fagocitário, provocado pela resposta do organismo confirmado na resposta imunitária detectada precocemente no $4^{\circ}$ DPI. Apesar da alterações macroscópicas observadas, o perfil bioquímico não acusou alterações que viessem a denunciar um comprometimento mais profundo nestes sistemas. Esses achados concordam com os apresentados por Bresciani (1997) e Svoboda et al. (1998) que observaram alterações semelhantes nesta fase da infecção.

Vidotto et al. (1987), Navarro et al. (1992) e Bresciani (1997), estudando várias espécies animais inclusive os cães, também demonstraram esses isolamentos, denunciando o perigo desses tecidos quando oriundo de animais para consumo humano.

Os achados histopatológicos (Quadro 2) mostraram alterações em cérebro, rim, linfonodo, pulmão, fígado e baço. Quando confrontados com os achados anatomo- 
patológicos e reisolamento demonstraram a patogenicidade do parasito nesses animais, apesar de não ter sido visualizado em nenhum tecido no exame direto. Esses resultados estão de acordo as observações feitas por, Svoboda et al (1998) e Bresciani (1997).

\section{Conclusão}

Todos os cães responderam ao estímulo antigênico, produzindo anticorpos anti-T. gondii.

Apresentaram alterações sorológicas, hematimétrica e bioquímica, demonstrando a importância desses achados. Da mesma forma que as alterações oftalmológicas, os achados de parasitemia e antomo-histopatológico confirmaram as observações indiretas, reforçando assim a importância desse parasito em cães jovens, como causa primária da doença.

\section{Referências}

AVERRIL, D.R.; LAHUNTA, A.de. Toxoplasmosis of The Canine Nervous System: Clinicopathological Findings in Four Cases. J.A.V.M.A., v.159, p.1134-1141, 1971.

BÓBIC, B. et al. Epidemiological relation ship betwen human toxoplasma infection and cats in Belgrade. Acta Vet Beograde, v. 45, n .2-3, p. 155-160, 1996.

BRESCIANI, K.D.S. Toxoplasmose Experimental em cadelas gestantes. 1997. 113 p. Dissertação (Mestrado) Univ. Est. Paulista.

BROOKS, K.D. Feline toxoplasmosis and human health. Veterinary Technician, v. 13, n. 8, p. 563-568, 1992.

CAMARGO, M.E. Improved technique of indirect immunuofluorescence for Serological diagnosis of toxoplasmosis. Ver. Inst Med. Trop., São Paulo, v. 6, n. 3, p. 117-118, 1964.

COSTA, A.J. et al. Experimental infection of bovines with oocyts of Toxoplasma gondii. J. Parasitology. v. 63, n. 2, p. 212-218, 1977.

DRESSEN, D.W. Toxoplasma gondii. J Am Vet Assoc, v. 196, n .2, p. 274- 276, 1990.

DUBEY, J. P. Toxoplasma, Neospora, Sarcocystis and other tissue cyst- forming of human and animals. In: KRIER, J.P. (Coord.) Parasitic Protozoa 2. ed. San Diego: Academic Press,. p. 1-157, 1993

DUBEY, J.P. Duration of immunity to shedding of Toxoplasma gondii oocystis by cats. J.Parasitology, v. 81, n. 3, p. 410-415, 1995.

FIALHO, S.A. Toxoplasmose Ocular. Contribuição ao Estudo Clínico e Experimental. 1953. Dissertação (Mestrado) - Fac. Nac.Med. Univ. do Brasil, 1953.

GERMANO, P.M.L.; ERBOLATO, E.B.; ISHIZUKA, M.M. Estudo sorológico da toxoplasmose canina, pela prova de imunuofluorescência indireta na cidade de Campinas. Ver Fac Med Vet, v. 22, p. 53-8, 1985.

GUIMARÃES, A.M.; RIBEIRO, M.F.B.; LIMA, J.D. Frequência de anticorpos anti-Toxoplasma gondii em cães de Belo Horizonte, MG. Arq Bras Med Vet Zootec, v. 44, n. 1, p. 6768, 1992.

HELLEY, D.M. Toxoplasmosis. J.Small Anim. Pract. v. 4, p. 627-629, 1970 .
HIRT, H. Toxoplasmosis. 2. ed. Buenos Aires: El Ateneo, 1976. $234 \mathrm{p}$.

ISHIZUKA, M.M.; MIGUEL, O .; BROGLIATO, D.F. Prevalência de Anticorpos anti- Toxoplasma em soro de cães no município de São Paulo. Rev. Fac. Med. Vet Zootec., v. 11, p. 115-125, 1974.

ISHIZUKA, M.M.; MIGUEL, O.; BROGLIATO, D.F. Incidência de Infecção por Toxoplasma gondii em cães do município de São Paulo. Ver. Fac. Med. Vet. Zootec., USP, v.18, p. 161-165, 1981.

JACKSON, M.H.; HUTCHISON, W.M. The prevalence and source of Toxoplama infection in the enviroment. In: BAKER, J.R.;MULLER,R. Advances in Parasitology. San Diego: Academic Press, v. 28, p. 55-105,1989.

JACOBS, L.; MELTON, M.L. A procedure for testing meat samples for Toxoplasma with preliminar results of a survey of pork and beef sample. J. Parasitol., v. 42, n. 2, p. 38-39, 1957.

KANEKO,J.J. Clinical biochemistry of domestic animals. V.II, Academic Press, 1989. p. 352.

KOESTNER, A.; COLE, C.R.; Neurophatology of canine toxoplasmosis. Am. J. Vet. Res., v.21, p.831-844, 1960.

LINDSAY, D.S. et al. Mechanical transmission of Toxoplasma gondii oocysts by dogs. Veterinary Parasitology., v.73, p. 27-33, 1997

McCABE, R.E. et al. Clinical spectrum in 107 cases of toxoplasmic lymphadenopathy. Ver. Infect. Dis., v. 9, p. 75474, 1987.

MERIC, S. M. Encefalite / Mielite / Meningite. IN: NELSON, R. W.; COUTO, C. G. Fundamentos de Medicina Interna de Pequenos Animais. Rio de Janeiro: Guanabara Kooogan, 1994. Parte 9, c. 70, p.566-571.

NAVARRO, I.T. et al. Estudo da resistência do Toxoplasma gondii ao efeito do cloreto de sódio e condimentos em lingüiça frescal de suínos. Boletim Sanit Panam, v. 112, p. 138-143, 1992.

NELSON, R.W.; COUTO, C.G. Fundamentos de Medicina Interna de Pequenos Animais. Rio de Janeiro: Guanabara Kooogan, 1994. Distúrbios Neuromuscu-lares: p.569-570.

NESBIT, J.W.; LOORENS, DC.; WILLIAMS, M.C. Spatic paresis in two Littermate pups caused by Toxoplasma gondii. J. S. Afr. Veter. Assoc., v. 52, p. 243-246, 1981.

NICOLLE, C.; MANCEAUX, L. Sur une protozoaire nouveau du gondi, Toxoplasma. Arch. Inst. Pasteur. Tunis., v. 2, p. 216-218, 1909.

OPPERMANN, W.H. Versuche zun experimentellen infection des hundes mit Toxoplasma- oozysten. 31p. 1971. Dissertation (Inaugural) - Universitat Berlin, Inst. Fur Parasitollogie des Fachbereiches Veterinarmedizin, Berlin.

REMINGTON, J.S.; DESMONTS, G. Toxoplasmosis. In: REMINGTON, J.S.; KLEIN, J.O. Infection diseases of the fetus and newborn infant. 2. ed. Philadelphia, Saunders, p. $142-253,1983$.

ROTH, J.A. et al. Fatal ocurrent Toxoplasmosis in a patient initially infected via a leucocyte transfusion. Amer. J. Clin. Path., v. 56, p. 601-605, 1971.

SABIN, A.B. Toxoplasmia encephalitis in children. J. Am. Med. Assoc., v.116, p.801-807, 1941. 
SANCHIS, F.S. Estudo da Reinfecção Experimental do gato pelo Toxoplasma gondii . 1978. Tese (Livre Docente) Fac.e de Med. Vet. e Zootecnia da Universidade Estadual de São Paulo, São Paulo, 1978.

SHARMA, S.P.; GAUTAM,O .P.; KHAROLE, M.U. Studies on some aspects of pathogenenis, chemotherapy and serology of experimental toxoplasmosis in dogs. Indian.Vet.J., v. 50, n. 7, p. 623-626, 1973.

SILVEIRA, C.A.M. Estudo da Toxoplasmose ocular na região de Erechin- $R S$, 1997. Tese (Doutorado) - Escola Paulista de Medicina).

SOUZA, W.J.S. et al. Epidemiological aspects of toxoplasmosis in Shoolchildren residing in localities with urban or rural caracteristics Within the city of Rio de Janeiro, Brazil. Mem.Inst.Oswaldo Cruz, v. 82, n. 4, p. 475-482, 1987.

SUTER, M. et al. Polymyositis-Plyradiculits Due to
Toxoplasmosis in the dog: Serology and Tissue Biopsy as Diagnostic Aids. Zentralblatt fur Veterinarmedizina, v. 31, n. 10, p. 792-798, 1984.

SVOBODA, M. et al. Postmortal Diagnosis os Toxoplasmosis in cats and dogs. Acta.Vet. Brno, v. 57, p. 31-38, 1998.

VIDOTTO, O. et al. Toxoplasmose experimental em porcas gestantes I. Observações clínicas e hematológicas. Arq. Bras. Med. Vet., v. 39, n. 4, p. 623-39, 1987.

VIDOTTO, O. et al. Estudos Epidemiológicos da toxoplasmose em suínos na região de Londrina-PR. Semina, v. 11, n. 1, p. 53-59, 1990.

VITOR, R.W.A.; PINTO, J.B. Eliminação de Toxoplasma gondii através de Urina, saliva e leite de caprinos experimentalmente infectados. Arq. Bras.Med.Vet. Zootec., v. 42, n. 2, p. 147-154, 1991. 